\title{
Memorias para optar al Título de Psicólogo que aportan al trabajo psicoterapéutico. Universidad de Chile 1994 a 1998
}

\author{
Laura MoncadaArroyo ${ }^{1}$,Andrés Costas Moreno ${ }^{2}$, Pedro Olivos Balmaceda ${ }^{3}$
}

\begin{abstract}
Resumen
El presente artículo muestra algunos de los resultados encontrados en la revisión de las memorias para optar al titulo de Psicólogo de los alumnos de Psicología de la Universidad de Chile realizadas entre los años 1994 y 1998 que aportan al quehacer psicoterapéutico, como un intento de sistematización que permita consolidar lo que se investiga.

Se presenta aquíla información obtenida en la revisión de las memorias, en relación a las siguientes unidades de análisis: marcos teóricos, tipo de estudio realizado, grupo etáreo y género.

Se encontraron creativas propuestas, destacándose algunas intervenciones diseñadas para trabajos individuales y grupales en diferentes lineas psicoterapéuticas. Se reseñan y destacan las memorias más originales.
\end{abstract}

\section{Abstract}

This article shows the results of a comprehensive review of the thesis done by undergraduate's students in order to obtain their title of Psychologist in the University of Chile between the years 1994 to 1998 . The review is focused in the thesis related to psychotherapy.

The theoretical frames, type of study, age and gender of the population and the contribution of the thesis are described.

Creatives psycotherapeutical interventions were found, including individual as well as group therapy in different psychological approaches. Originals works are emphasized.

Key words: thesis, investigations, and psychotherapy findings

\section{Introducción}

La investigación en la Universidad es una tarea fundamental. Aunque se realizan más investigaciones a nivel de post grado, estimulado esto por algunos concursos que circulan en nuestro país, también es necesario reconocer lo que hacen los alumnos de pregrado en este ámbito.

En el campo de la psicología chilena nos encontramos con la realidad que la mayoría de las Escue-

\footnotetext{
${ }^{1}$ Psicóloga Clinica y Supervisora Acreditada, Docente del Equipo de Psicoterapia, Departamento de Psicología, Universidad deChile.email:1moncada@usa.net

${ }^{2}$ Licenciado en Psicologia, Ayudante del equipo de Psicoterapia, Departamento de Psicologí, Universidad de Chile.

${ }^{3}$ Estudiante en Práctica, Ayudante del equipo de Psicoterapia, Departamento de Psicología, Universidad de Chile.
} 
las de Psicología incluyen dentro de la formación del psicólogo una memoria para optar al título que tiene altas exigencias metodológicas.

Ocurre entonces que los alumnos de pregrado, se ven enfrentados a la tarea de investigar sobre variados temas, haciendo muy buenos aportes, tal como lo comprobamos en un artículo anterior. (1)

Algunos alumnos que se sienten atraídos por el área clinica, optan por hacer diferentes contribuciones durante su trabajo final de pregrado, tal como investigar sobre cuestionarios, estandarizar un test, o comprobar la eficacia de algunas intervenciones psicoterapéuticas.

De todos estos esfuerzos de pregrado, han resultado muchos trabajos excelentes que son muy poco difundidos, lo que fue la principal motivación para emprender este estudio.

Ya en la revisión de las Memorias para optar al título de Psicólogo del artículo de 1997 antes mencionado, nos encontramos con aportes muy valiosos para la práctica de la psicología clínica en nuestro medio, en las distintas orientaciones psicoterapéuticas. Esto nos motivó a volver a mirar lo que ha ocurrido en los años siguientes, en este caso sólo de los trabajos hechos en la Universidad de Chile, y rescatar los nuevos hallazgos.

Para este estudio utilizaremos como definición de psicoterapia: "un proceso de interacción emocional y cognitivo principalmente verbal, pero también no-verbal, consciente y planificado, cuyo objetivo es modificar trastornos conductuales y estados de sufrimiento psíquicos; este proceso se realiza dentro de un consenso -en lo posible, entre el terapeuta, el paciente y su grupo de referencia -y está destinado a personas necesitadas de tratamiento. El proceso psicoterapéutico se desarrolla en la dirección de una meta elaborada y definida, meta que, en lo posible, debe ser común a los involucrados en él (disminución de sintomas y/o cambio estructural de la personalidad). La psicoterapia se lleva a cabo por medio de técnicas susceptibles de ser aprehendidas sobre la base de una teoria de la conducta normal y patológica. Este proceso se apoya, de regla, en una relación emocional entre los parti- cipantes" (2). Y, como aporte al trabajo psicoterapéutico vamos a entender desde las reflexiones epistemológicas en torno a la psicoterapia y quienes la practican, hasta la aplicación de tests y técnicas que pueden ser útiles a la consecusión del fin acordado entre el terapeuta y el paciente en el contexto terapéutico.

\section{ElEstudio}

Nuestro estudio abarca el universo de todas las memorias del Departamento de Psicología de la Universidad de Chile que se realizaron entre los años 1994 y 1998 para optar al título de Psicólogo.

En dicho periodo se encontraron 139 memorias, de las cuales 60 tienen relación con la práctica clínica. Sin embargo, la muestra de este estudio está compuesta por 57 memorias, ya que éstas son las que están efectivamente disponibles en la biblioteca de la Facultad de Ciencias Sociales de la Universidad de Chile .

Tal distinción se llevó a cabo teniendo como principal criterio el título de la investigación, en razón que, metodológicamente se sugiere que éste sea una descripción del contenido del estudio: "debe ser conciso y específico, y, en lo posible, nombrar expresamente las variables principales o dimensiones de esta" (3).

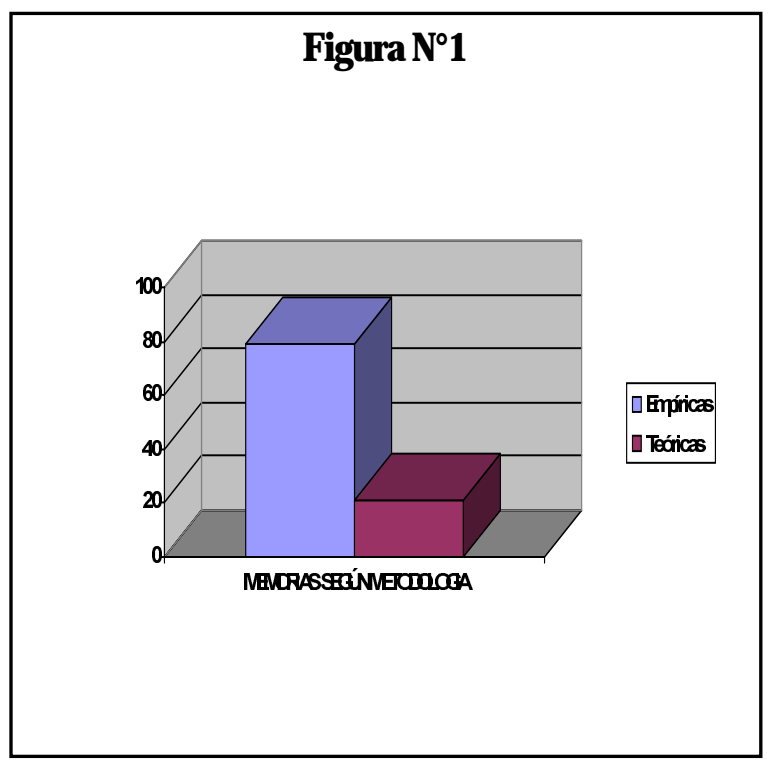


De este modo, el titulo nos permitió distinguir las investigaciones en psicoterapia de investigaciones realizadas en otras áreas de la psicologia. Cuando el título no aportaba información suficiente para distinguir con claridad su categorización, se recurrió a la fuente directa para dirimir la situación.

Una vez clasificada la muestra de memorias vinculadas con el quehacer psicoterapéutico se procedió a una lectura y análisis completo de estas. A partir de dicho análisis se establecieron las categorías que nos permitieron organizar la información recogida.

\section{Memorias según su Metodologia}

Las memorias vinculadas a la práctica psicoterapéutica se clasificaron según si el estudio era Empírico o Teórico. Los resultados obtenidos indican un total de 45 memorias empiricas, equivalentes a un 79\%; y de 12 memorias teóricas, correspondientes al $21 \%$ restante.

Considerando el total de memorias empíricas, estas se clasificaron a su vez, según el tipo de investigación que realizan. En la mayoria de los casos esto es explicado por los propios autores al describir los aspectos metodológicos de sus investigaciones, sin embargo, en ciertos casos en que esto no ocurrió, la clasificación se llevó a cabo considerando el procedimiento, los objetivos y las conclusiones de la investigación.

Los resultados que se obtuvieron señalan que existen 6 categorias de Tipo de Investigación. Estas son: Exploratorio (7\%), Descriptivo (11\%), Exploratorio-descriptivo (43\%), Descriptivocorrelacional (33\%), Exploratorio-Descriptivocorrelacional (2\%) y Explicativo (4\%).

\section{Tiposdeintervención}

Además, las memorias empíricas fueron analizadas según sí su aporte a la práctica Clínica se enmarca en lo: Individual, Diádico, Grupal, Institucional, o Mixto.

Los resultados señalan que 19 memorias (42\%) apuntan como foco de su intervención a la atención individualizada de sujetos. Estas memorias, se abocan, a través de cuestionarios (Por ejemplo: Ref. $\mathrm{N}^{\circ} 10$ ), pruebas psicométricas (ejemplo: Ref. $\mathrm{N}^{\circ} 19$ y 31) e intervenciones psicológicas en el marco de la terapia (ejemplo: Ref. $N^{\circ} 29$ ), a medir, describir $\mathrm{y} / \mathrm{o}$ intervenir sobre dimensiones psicológicas individuales. (Ver nómina de estudios revisados en apéndice)

Por otro lado, un 36\% de las memorias empiricas (16 de ellas), apuntan a la medición, descripción y/o intervención sobre estructuras y/o procesos grupales (ejemplo: Ref. $N^{\circ} 15,25,38$, 43 y 50). Mientras que 2 de las memorias empiricas que aportan a la práctica clínica (4\%) se focalizan sobre intervenciones a las que hemos denominado institucionales. Estas corresponden a la descripción y el análisis de los procesos diagnósticos en una institución psiquiátrica (Ref. $\left.\mathrm{N}^{\circ} 30\right)$ y a la definición del rol del psicólogo en un equipo multidisciplinario en un centro de atención para pacientes con Accidentes cerebro-vascular (Ref. $\left.\mathrm{N}^{\circ} 18\right)$.

Así mismo, encontramos 1 memoria (2\%) que presenta un modelo de trabajo diádico, ya que fija su objetivo en la intervención sobre los patrones de vinculación con sus madres, que presentan las menores victimas de abusos incestuosos (Ref. $\mathrm{N}^{\circ} 48$ ).

Por último, se encuentran 7 memorias (16\%) a las que consideramos mixtas, ya que sus planteamientos pueden dirigirse tanto a lo individual, diádico y/o grupal (Ejemplo: Ref. No 20, 49 y 55). Lo señalado anteriormente puede verse en la Figura $\mathrm{N}^{\circ} 2$. 
Figura $\mathrm{N}^{\circ} 2$

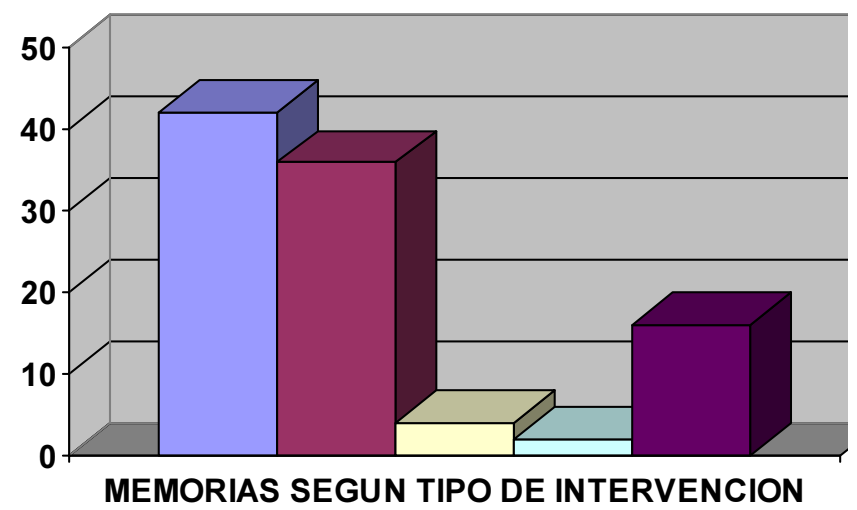

\section{Memorias segúnsu marco Teórico}

Un aspecto importante en el desarrollo de este estudio tiene relación con los marcos teóricos que fundamentaron la aplicación empírica de una metodologia, un programa de intervención o un instrumento de medición psicológica en el quehacer de la psicología clinica. Por otro lado, se destaca el trabajo de indagación bibliográfica sobre el desarrollo de conceptos teóricos propios de la disciplina. El análisis de las memorias nos permitió clasificar sus marcos teóricos según los cuatro enfoques psicoterapéuticos impartidos en la actual malla curricular del Departamento de Psicologia de la Universidad de Chile, (a saber: enfoque CognitivoConductual, enfoque Humanista-Experiencial, enfoque Psicoanalitico y enfoque Sistémico), estableciendo además una categoría de orientación teórica mixta y otra, que agrupa otros modelos teóricos distintos.

Dentro de los cuatro enfoques mencionados, el enfoque Sistémico se encuentra como marco conceptual en 11 de las memorias (19\%) que aportan a la práctica clínica (ejemplo: Ref. No 14, 34,35, 39y 57 ).

El enfoque Cognitivo-Conductual es citado como marco principal en 6 de las memorias revisa- das, lo cual corresponde al 11\% del total (ejemplo: Ref. $N^{\circ} 1,11$ y 26). El Psicoanálisis sirve como base teórica a 5 de las memorias seleccionadas, equivalentes al 9\%. (Ejemplo Ref. N 7, 8, 33 y 54 ).

El enfoque Humanista-Experiencial se presenta como base conceptual preponderante en 4 de las memorias revisadas para este estudio. Dicho número corresponde al $7 \%$ del total de memorias seleccionadas (ejemplo: Ref. $\mathrm{N}^{\circ} 16,29$ y 55 ).

Tras la revisión de las memorias seleccionadas, se definió un enfoque teórico mixto como aquel que combina conceptos de diferentes corrientes teóricas fundamentales con otros modelos conceptuales como la Psicologia de la Salud y Psicología del Desarrollo, por ejemplo. En esta condición, encontramos 18 Memorias, lo que equivale al $31 \%$ del total de ellas. (Ejemplo: $\mathrm{N}^{\circ}$ 4, 9, 22, 43 y 48).

Por último, se estableció una categoria "Otra" que agrupa a aquellos marcos teóricos que aparecen como independientes de los modelos teóricos de los enfoques que hemos citado.

Las memorias clasificadas de este modo son 13, correspondientes al 23\% del total (Ejemplo: Ref. $\mathrm{N}^{\circ}$ 6, 47 y 56). Lo anteriormente señalado se observa en la Figura $N^{\circ} 3$. 


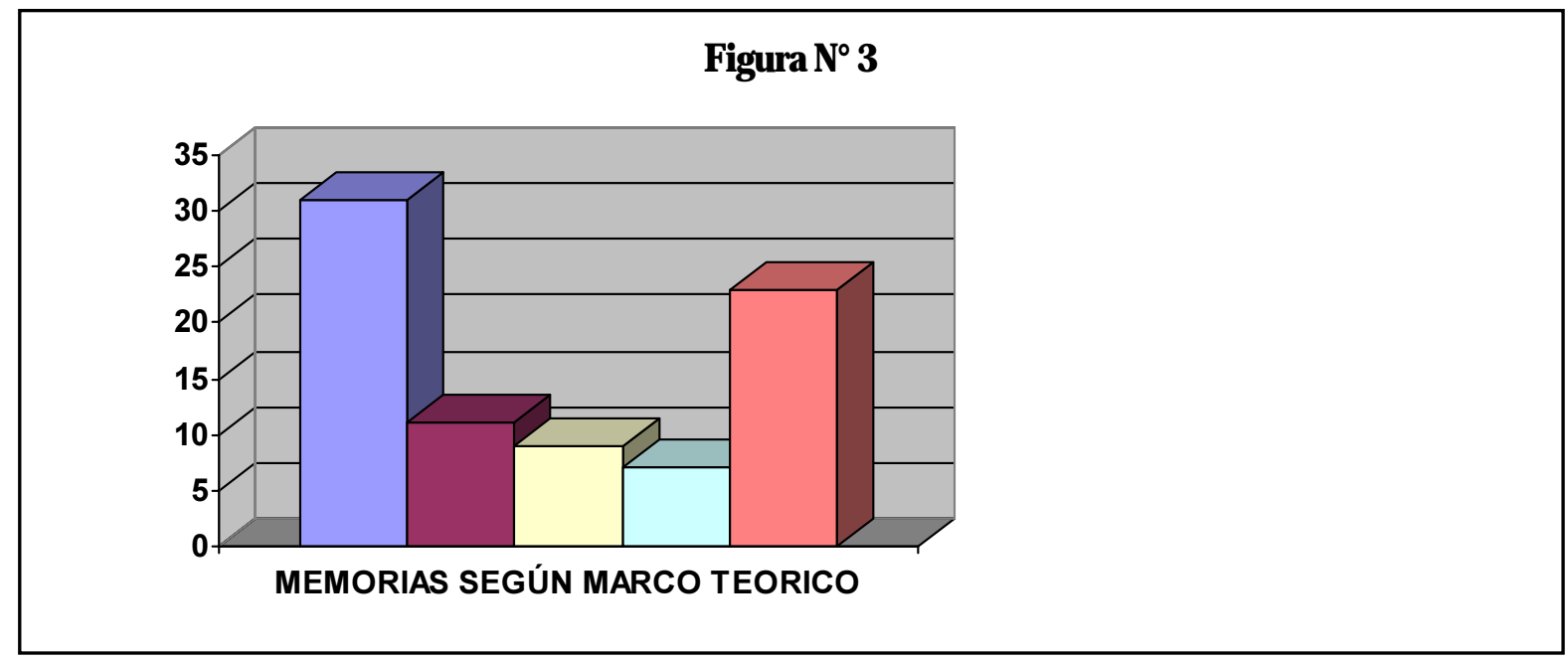

\section{Grupo Etareo como Unidad de Análisis}

Otro aspecto de interés fue el grupo etáreo al que se dirigieron las memorias seleccionadas. Dicha clasificación se efectuó a través de la revisión de las muestras que se utilizaron en cada memoria, suponiendo que la aplicación de la metodología que se presenta puede ser extrapolada a otras muestras dentro del mismo rango de edad. Además, caben también aquí, memorias que siendo teóricas no utilizaron muestras especificas, pero que sin embargo, están dirigidas a un grupo etáreo determinado.

Las categorias de análisis fueron dadas por Pappalia y Olds (4), quienes distinguen los siguien- tes grupos etáreos en forma amplia: Infancia (0 - 12 años), Adolescencia (13 - 20 años), Adulto (21 - 65 años), Adulto mayor (65 años en adelante). Además, se estableció la categoria de Mixta para aquellas memorias cuyos objetivos atraviesan diferentes grupos etáreos, y la de Sin Especificar, para aquellas que no presentan información al respecto.

La clasificación se estructura, entonces, del siguiente modo: 9 Memorias (16\%) que tienen como objeto de estudio niños (ejemplo: Ref. $\mathrm{N}^{\circ} 21$ y 49 ), 10 Memorias $(18 \%)$ cuyos sujetos eran adolescentes (ejemplo: Ref. $\left.\mathrm{N}^{\circ} 5 \mathrm{y} 37\right), 7$ Memorias (12\%) con Adultos (ejemplo: Ref. $\mathrm{N}^{\circ} 18$ y 36), 22 Memorias Mixtas (38\%) (ejemplo: Ref. $\mathrm{N}^{\circ} 15$ y 53) y 9 Memorias Sin especificar (16\%) (Ejemplo: Ref. N ${ }^{\circ} 33$ y 52).

\section{Figura $\mathrm{N}^{\circ} 4$}

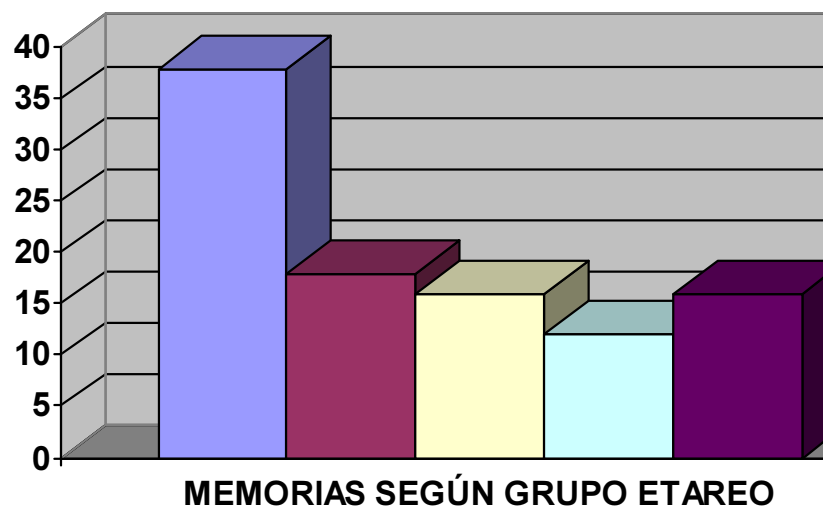




\section{Género como Unidad de Análisis}

Finalmente, se llevó a cabo una clasificación de las memorias seleccionadas, tomando en cuenta el género de sus muestras. De esta manera, 11 memorias $(19 \%)$ se enfocan al trabajo con mujeres (ejemplo: Ref. $\mathrm{N}^{\circ}$ 9, 29 y 48), 5 al trabajo con hombres
(9\%) (ejemplo: Ref. $\mathrm{N}^{\circ} 21$ y 50 ), 32 memorias (56\%) se clasifican como mixtas (dirigidas tanto a hombres como a mujeres) (Ejemplo: . N 4 y 56 ) y 9 memorias (16\%) Sin Especificar, que no presentan información al respecto (Ejemplo: Ref. $\mathrm{N}^{\circ} 8$ y 33 ).

Esta clasificación de las Memorias, es graficada en la Figura $\mathrm{N}^{\circ} 5$.

\section{Figura $\mathrm{N}^{\circ} 5$}

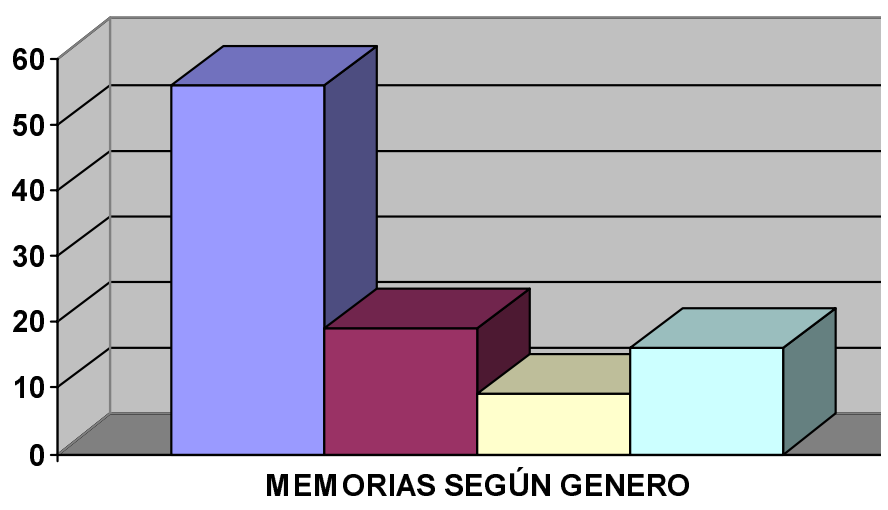

\section{Comentarios Finales}

Deseamos resaltar luego de esta revisión, dos tipos de reflexiones; una reflexión global y varias específicas a nivel más pragmático.

La psicología clínica de fines de siglo -más allá de la discusión sobre las metodologías adecuadas en ciencias sociales- necesita nutrirse de los hallazgos de las investigaciones para perfeccionar su práctica terapéutica. Todos los esfuerzos que vayan en esta dirección son interesantes, y en el contexto académico chileno es útil conocer todos los trabajos que existen. "La evidencia sugiere que los clínicos tienden a subestimar el requisito de informarse y cuando reconocen que necesitan información, ellos no acceden a las fuentes con menos sesgo y más confiables. Esto conduce a que exista una brecha entre la investigación y la práctica clínica, lo que se traduce en una práctica clínica poco respaldada. Hay dos explicaciones para esta variante: 0 lo que ocurre es que no hay evidencias en las cuales la práctica clínica puede basarse, o si existe, pero al menos algunos de nosotros no la usamos. El resultado inevitable de esto es que algunos de nuestros pacientes no están recibiendo el mejor cuidado que existe" (5).

Las reflexiones que nos parece importante destacar en un nivel más específico son, por un lado, las similitudes con el estudio anterior-que en este caso fueron dos- y por otro, temáticas sugerentes durante este período.

En relación a las similitudes, el grupo adulto mayor en el presente trabajo no tuvo representación, mientras que en el anterior fue de un $1 \%$. Nos parece lamentable que así sea. Este grupo etáreo es un nuevo desafio para la psicologia de fin de siglo en todos los continentes. En Chile, actualmente los adultos mayores tienen nuevos espacios donde participan colectivamente en actividades, por ejemplo en Municipalidades, donde se han creado progra- 
mas que los incluyen, lugares donde podrian ser abordados para su inclusión en muestras de estudios.

También ocurre en los trabajos de éste periodo, al revisar la variable género, que los hombres participaron menos proporcionalmente en las investigaciones, como se detectó en el estudio de 1997.

Probablemente, si fueran abordados en sus lugares de trabajo, podrian aumentar estas estadísticas, con toda la riqueza que ello puede significar.

Además, deseamos destacar que de nuestra muestra, un $79 \%$ de los trabajos realizados utilizaron una metodología empírica, lo que implica que un alto porcentaje de los estudiantes interactuaron con distintos sectores de la población, como investigadores activos, lo que contribuye, a nuestro juicio, a su formación como profesionales.

Asi también, encontramos notables aportes dentro de las memorias teóricas, dentro de las cuales encontramos una linea de investigación en la linea psicoanalítica, que aporta tanto a la vida académica como al ejercicio psicoterapéutico (Memorias $\mathrm{N}^{\circ}$ $7,8,33$ y 54$)$.

Otra líneas de investigación muy importantes encontradas, son las que se realizaron en torno al tema de la hipnosis Ericksoniana, que en este período revisado fue orientado a diferentes muestras ( Tesis $\mathrm{N}^{\circ} 16,27,40$ y 42 ); y en torno al test de Rorschach donde fueron estudiados cuadros médicos y psicopatológicos tales como : anorexia nerviosa, hemodializados, cáncer cérvico-uterino, trastorno bipolary adictos a pasta base y cocaína. (Tesis $^{\circ} 2,4,9,31$ y 56 ), todas con resultados muy sugerentes.

Por otro lado, en un tema emergente como ha sido el de la violencia intrafamiliar se encontraron numerosos trabajos. Así, memorias en relación a este tema, con muestras mixtas de niños maltratados son las $\mathrm{N}^{\circ} 1$ y 11 , con muestras de adolescentes son las $\mathrm{N}^{\circ} 22$ y 48 , con muestras de mujeres $\mathrm{N}^{\circ} 49$ y 51 y aporte teórico en el tema $\mathrm{N}^{\circ} 46$.

Temas novedosos que no habian aparecido en elartículo que publicáramos en 1997, son el exilio y retorno, la creatividad, la intencionalidad suicida, y rasgos de personalidad de la trabajadora sexual. (Tesis $\mathrm{N}^{\circ} 6,12,15 \mathrm{y} 36$ )

Por razones obvias de espacio no podemos destacar todas las memorias que quisiéramos, pero mencionaremos algunas que nos parecen extraordinarios esfuerzos, y contundentes aportes tanto a la vida académica como al ejercicio de la psicología clinica.

Ellas son: (Se las cita con el número en que se encuentran en el Apéndice).

\section{a) MemoriasTeóricas:}

(7) Avendaño: Este trabajo analiza la transferencia, la contratransferencia, la atención parejamente flotante y la interpretación en la obra de Freud como problemas epistemológicos.

(44). Kaplán: Diseña un programa de desarrollo emocional-social para niños entre cuatro y seis años articulando material relacionado con el autoconocimiento, autoestima y relaciones sociales en estas edades. Esta memoria constituye un aporte al sistema de educación pre-básica y a la Psicología clínica infantil.

(52) Piola: Este trabajo recopila y sistematiza mucha información respecto de los enfoques terapéuticos de disfunciones sexuales, cumpliendo plenamente con lo prometido en sus planteamientos iniciales. Revisa los siguientes autores: Masters y Johnson, H, Singer Kaplan, Lo Piccolo, Zilbergeld, Rinkleib, Hartman, Fithian, Gilhot y Letuve, Lazarus y Ellis. Además hace una revisión de varias décadas de investigaciones tanto en el extranjero como nacionales en relación a las disfunciones sexuales. Por último hace un análisis comparativo de varios enfoques y analiza los aportes y limitaciones de cada uno.

(57) Zamorano: Este trabajo hace una revisión y sistematización extensa de los antecedentes teóricos de la Biología del Conocer y la Terapia Familiar. Aborda en qué consiste un motivo de consulta familiar, cómo se constituye el sufrimiento que lleva a la familia a consultar y especifica en que consiste el cambio terapéutico. 


\section{b) Memorias Empíricas:}

(6) Arqueros: Esta memoria es un intento original de medir el impacto de las experiencias del exilio y el retorno a través del Test de Rorscharch. A través de la aplicación y los resultados obtenidos por medio de esta herramienta clínica, se enriquece el trabajo terapéutico con los retornados.

(20) Córdova y Mc Pherson: En esta memoria se plantea una interesante aproximación a la comprensión de la elección de pareja desde una mirada de la sociología, la corriente psicoanalítica y el enfoque cognitivo. Luego de hacer análisis valiosos con sus hallazgos en ocho parejas, dejan planteadas reflexiones en torno a preguntas que son de gran interés para los psicólogos clínicos, debidamente sistematizadas.

(24) Cruzaty Astorga: Estos autores logran una buena revisión de la perspectiva multigeneracional en el desarrollo de la Teoría Familiar Sistémica. A su vez, diseñaron un taller y dos instrumentos de medición (Diferenciación del Sí mismo y Facilitación del proceso de Diferenciación) utilizados con una muestra de doce personas. Los participantes reportan cambios personales y el taller satisfizo los objetivos propuestos.

(29) Elgueta: Si bien este trabajo fue aplicado sólo a dos casos de madres primigestas, entrega mucho material respecto del apoyo psicológico temprano de ellas, tanto para los académicos, como para los psicoterapeutas. Se presentan en forma ordenada las sesiones completas realizadas con las pacientes. Además crea un cuestionario de auto-reporte para evaluar la terapia. Las técnicas usadas (Focusing de Gendlin, Reflejos de Rogers y Técnicas Ericksonianas y de Rossi) son clasificadas y explicadas en los anexos correspondientes.

(31) Filippi: Un aporte empírico al estudio y la definición del concepto de trastorno bipolar es lo central de este trabajo. Su procedimiento es cuidadoso, estableciendo un paralelo entre lo clínico y lo psicométrico. Su discusión es sistemática y bien articulada.

(39) Greve, Molinay Quiroz: En este trabajo las autoras muestran una intervención grupal en un tema tan importante como es el rendimiento académico. Presentan una metodología que logra involucrar a los veinte participantes y despertar en ellos recursos nuevos de gran utilidad y eficacia para su vida universitaria.

(40) Guzmán: La propuesta terapéutica de este trabajo (utilización de la hipnoterapia en la resolución de sintomatología asociada al abuso sexual) resulta novedosa en nuestro medio. Se hace una descripción detallada de la terapia estratégica e hipnótica utilizada, la que reveló ser útily efectiva. Es interesante destacar que el formato de tratamiento propuesto es igualmente válido para cualquier causal de estrés post-traumático, sólo con la salvedad de cambiar los contenidos.

(45) Labarthe: Este trabajo comprueba la eficacia de la terapia cognitiva-conductual en pacientes alcohólicos. La terapia interfiere la conducta de ingesta de alcohol aumentando la abstinencia.

Por último, continuamos convencidos, tal como lo dijéramos en el artículo anterior, que el investigar como un requisito para recibir el titulo es una instancia privilegiada, puesto que la realización de una memoria y su defensa ante una comisión evaluadora-sin que se excluya además una práctica profesional- otorgan una formación más sólida a nuestrosalumnos.

\section{Referencias}

1. Moncada L, Parra M, Lara S . Análisis de las tesis para optar al título de psicólogo en el área de la Psicoterapia en cuatro universidades chilenas en el período 1990-1995. Revista Terapia Psicológica, año XV, volumen VI (3), № 27, 1997

2. Strotzka (1975) en Jimenez P. Un modelo de análisis del sistema de atención psicoterapéutica en Investigación en Psicoterapia procesos y resultados. Society for Psychotherapy Research. Capitulo Latinoamericano. Corporación de Promoción Universitaria. Santiago de Chile, 1995

3. Kerlinger F. Investigación del Comportamiento. Editorial McGraw Hill, Mëxico, 1988.

4. Pappalia D, Olds S W. Desarrollo Humano. Editorial McGraw Hill, Santa Fe de Bogotá, Colombia 1993.

5. Geddes J. "Evidence based practice in Mental Health". Vol. 1 Number 1. BMJ Publishing Group, England. February, 1998. 


\section{Apéndice: Memorias Revisadas}

1.- Aburto Y. y OrellanaS. Estudio descriptivo del desarrollo afectivo social de niños de 6 a 11 años de edad, victimas de maltrato en el ámbito familiar, a través del Test de Apercepción Temática Infantil (CAT-H). 1996.

2.- Alvarez M. y TaboadaM. Características del Test de Rorscharch en Anorexia Nerviosa: Hacia un perfil de personalidad. 1994.

3.- Aranda, Brantey Robles. Estandarización de la Escala de Desarrollo Psicosocial de René Zazzo y Marie Claude Hurtig en niños de 5 años de edad. 1995.

4. -Aravena, O. y Bravo, A.Estudio Descriptivo de la personalidad a través del Test de Rorschach en pacientes hemodializados. 1996.

5. -Araya, O. y Nuñez, N. Estudio Descriptivo de las características de una consulta en las áreas de sexualidad, reproducción y anticoncepción, realizada por adolescentes entre 13 y 18 años de nivel socio económico bajo, en cinco consultorios de la Región Metropolitana. 1994.

6. -Arqueros, J acqueline. Exilio y Retorno abordados desde Rorschach. 1995.

7. -Avendaño, Rodrigo. Epistemologia de la técnica Freudiana. 1997.

8. -Banderas, J aviera. Contribución al análisis del concepto de inconsciente en la obra de Sigmund Freud. 1996.

9. -Biason, L. y Vöhringer, C. Estudio Descriptivo - Exploratorio de rasgos de personalidad en pacientes con cáncer cérvico - uterino a través del Test de Rorschach. 1997.

10. -Bofill, J orge. Elaboración de un Cuestionario acerca de tres funciones del Yo, como indicadores de adecuación de la elección vocacional, de acuerdo con la teoría Ginzberg. 1996.

11. -Bruzzo, R. y Reveco, M. Evaluación del cambio en las variables Autoestima y Depresión en niños víctimas de maltrato familiar, del primer ciclo básico, producto de la aplicación de un programa de resolución de problemas interpersonales. 1997.

12. -Bustos, S. y Silva, A. Por sus obras los conoceréis. Reposicionamiento del concepto de creatividad en la Psicologia actual. 1998.

13. -Carbonell, Colodroy Molina. Proposición de intervenciones psicoterapéuticas para pacientes con trastorno de estrés post traumático atendidos en el hospital del Trabajador. 1997.

14. -Carlini, P. y Castro, E. Pautas de explotación relacional presentes en la dinámica familiar de las víctimas de incesto. Un estudio de casos. 1996.

15. -Christensen, Cynthia. Intencionalidad Suicida. 1995.
16. -Cobian, Patricia. Aportes de la Hipnoterapia Ericksoniana en el control y manejo del dolor agudo en el parto normal. 1995.

17. - Coddou, A. y Chadwick M. Evolución del concepto de codependencia. 1994.

18. -Contreras, Mãangélica. Estudio Exploratorio del abordaje psicológico en rehabilitación de pacientes con A.C.V. hacia una definición del rol del psicólogo. 1995.

19. -Contreras, Waldo. Estudio Exploratorio-descriptivo sobre indicadores emocionales en la prueba del dibujo de la figura humana de Koppitz, en niñas de un hogar de menores. 1997.

20. -Córdoba, C. y MacPherson, J . Influencia de la familia de origen en la elección de pareja. 1998.

21. -Correa, E. y Moyano, A. Estudio Exploratorio de las características emocionales que presenta un grupo de niños que cursa segundo y tercer año de la E.G.B. con trastornos específicos del aprendizaje en el ámbito escolar a través del Test de Apercepción Temática (TAT escolar) en un colegio de nivel socio económico medio perteneciente a la Región Metropolitana. 1995.

22. - Correa, A. y Riffo, M. Descripción y Análisis de la relación victima-autor en los delitos sexuales y su repercusión en la autoestima y el trastorno de estrés post traumático en mujeres adolescentes atendidas en el C.A.V.A.S. 1995.

23. -Cortés, J .M. y García, I. Relaciones entre pautas de interacción familiar y comportamiento escolar. 1996.

24. -Cruzat, A. y Astorga, A. Creación e implementación de un taller de trabajo con la propia familia de origen, a través del genograma, para modificar el grado de diferenciación del si mismo, de un grupo de estudiantes universitarios de la carrera de Psicologia de la Universidad de Chile. 1998.

25. -Decintri, M. y Fuentes, V. Desarrollo, aplicación y evaluación de un programa basado en el método de resolución de problemas para prevenir las conductas violentas en adolescentes. 1997.

26. -Díaz, M. y Pemjean, A. Aportes del Enfoque cognitivoconductual a la conceptualización del dolor crónico lumbar: revisión bibliográfica. 1996.

27. -Donoso, Renato. Evaluación experimental de tres estilos comunicacionales para impartir directivas terapéuticas en el tratamiento ortodóncico de niños con labio leporino y/o fisura vela palatina. 1995.

28. - Dunner, P. y Valenzuela, M. Adaptación y tipificación del Test DAP-SPED en niños de 10 a 14 años de edad en nivel socio económico alto, pertenecientes al área Metropolitana. 1996.

29. -Elgueta, Patricio. Una Intervención de apoyo psicológico temprano desde el enfoque experiencial en madres primigestas. 1996.

30. -Farías J y FerrarioA. Proceso Diagnóstico en la Clinica Psiquiátrica Universitaria. 1998. 
31. -Filippi, Giselle. Trastorno bipolar: un estudio de personalidad y su articulación con la patologia. 1996.

32. -Gaete, Gloria. Sistematización de la experiencia del programa de apoyo a jóvenes detenidos en el centro de detención preventiva en Puente Alto. 1995.

33. -Galaz, C. y Valdebenito, M. Los Perversos: La estructura de personalidad perversay la relación terapéutica. 1995.

34. -Gallero, P. El modelo estratégico breve como enfoque de la problemática adolescente. 1994.

35. -Gálvez, F. y Morales, M. Infidelidad en la pareja: ni victimas ni culpables: una perspectiva sistémica. 1998.

36. -Garzón, M. y Mella, C. Estudio Psicológico sobre rasgos de personalidad e indicadores patológicos de la trabajadora sexual. 1995.

37. -González, Carolina. Cambios en la percepción de la sexualidad en un grupo de adolescentes a través de un estudio antesdespués de un taller de sexualidad humana. 1994.

38. -González, R. y Rivas, R. Adaptación Psicosocial en pacientes epilépticos de clase social alta, baja y media. 1995.

39. -Greve, Molina y Quiroz. Aplicación del modelo sistémico estratégico breve en estudiantes universitarios con bajo rendimiento académico: un estudio experimental. 1994.

40. -Guzmán, Gonzalo. Utilización de la Hipnoterapia en la resolución de la sintomatologia asociada a abuso sexual: un estudio piloto. 1996.

41. -Hernández, I. y Lepe, J . Problema psicosomático: la dinámica del lenguaje dada en sistemas en los cuales uno de sus miembros presenta un cuadro sintomático. 1994.

42. -Herreros, M. y Venegas, A. Hipnosis en el manejo y control del dolor: una propuesta de intervención en pacientes oncológicos infantiles y juveniles con leucemia linfoblástica aguda. 1994.

43. -Ibarra, X. y Mendez, D. Estudio Exploratorio y Descriptivo de la estructura familiar de niños oncológicos atendidos en un centro hospitalario del área sur de Santiago. 1994.

44. Kaplan Patricia. Aporte al desarrollo del autoconocimiento de la autoestima y de las relaciones sociales a partir de un programa de estimulación para niños de 4 a 6 años en jardines infantiles. 1997.

45. -Labarthe, Antonio. Efectos de un programa de rehabilitación de orientación cognitiva conductual en un tratamiento farmacológico para pacientes alcohólicos. 1995.

46. -Lemelson deZ, Beatriz. Vinculos patológicos. La violencia familiar. 1996.

47. - Melo, P. y Sarrazín, C. Estudio descriptivo comparativo de las actitudes hacia la relación de pareja, el matrimonio y la convi- vencia en adolescentes provenientes de familias con padres separados y de familias intactas de nivel socio económico medio y exploración de cuatro variables de la dinámica familiar vinculadas a la relación de pareja. 1995.

48. -Navarro, Carolina. Patrones de vinculación en madres de victimas de abusos incestuosos: los peligros del vínculo. 1998.

49. -Olavarria, Rodrigo. Adaptación de la Escala de Madurez Mental Columbia en una muestra de niños que presentan déficits auditivos de 7 a 9 años de la Región Metropolitana. 1994.

50. -Ortiz, L. y Urrea, J . Investigación Metodológica - Exploratoria hacia la búsqueda de actitudes en hombres universitarios en relación al rol femenino, mediante estímulos proyectivos creados. 1995.

51. -Pérez, F. y Raurich, C. Subjetividad, Género y Violencia doméstica. 1996.

52. -Piola, Mạ-Gloria. Enfoques Terapéuticos de disfunciones sexuales masculinas y femeninas. 1994.

53. -Segura, C. y Valdivia, C. Estudio Exploratorio-DescriptivoComparativo a través del Test de Rorschach de las caracteristicas de personalidad en adolescentes diabéticos insulino - dependientes. 1995.

54. -Soto, Christian. Estudio sobre el trabajo Freudiano en torno ala melancolia. 1996.

55. -Thomsen Q., Marcela. Concordancia de Predicados: una reformulación del Manual de Focalización Experiencial y un análisis de su influencia en los niveles de experienciar. 1996.

56. -Visintini, Paola. Descripción de Personalidad de un grupo de jóvenes adictos a la pasta base de cocaina desde el Test de Rorschach. 1995.

57. -Zamorano, Claudio. Biología del conocer y terapia familiar. 1996. 